\title{
Study of Wavelet Transforms In De-Noising (Using Cauchy Distribution)
}

\author{
${ }^{1}$ Ashish Dhiman \\ ${ }^{1}$ Department of Electronics and Communication, Delhi Technological University (Formerly Delhi College of \\ Engineering), Delhi, India),
}

\begin{abstract}
Degraded signal has an adverse effect on the performance of the system and therefore it must be removed before further processing of signals. Spectral analysis through the Fourier transform is the simplest way to remove the noise, but it is limited to only stationary signals. Non stationary signals require continuous analysis of the signal in time and frequency domain. Wavelets provide better time, frequency localization and multi-resolution analysis compared to the Fourier transform. In this paper, we use the Cauchy probability density function for the study of different wavelets in denoising of audio signals. Compared to Gaussian Density Function, Cauchy distribution shown the non Gaussian statistics with heavy tailed distribution. Soft and Hard thresholding are estimated at each decomposition level and performance are evaluated in terms of signal-tonoise ratio $(S N R)$.
\end{abstract}

Keywords: wavelet, level of Decomposition, wavelet thresholding, Cauchy distribution, denoising, SNR

\section{INTRODUCTION}

A noise is an undesired random signal which deteriorates the quality of the original signal. Therefore, it must be removed without losing the original characteristics of the signal. The simplest method for removing the noise is filtered based Fourier transform[1] but such filtering methods are successful only when removing high frequency noise from low frequency signals. This method also alters the original signal characteristics and fails to give satisfactory results under real scenarios i.e. for non-stationary signals. The Short Time Fourier Transform (STFT)[2] overcomes this drawback by using the windowing technique in analyzing the frequency behavior of signal with time. But due to fixed window length STFT does not provide better time and frequency resolution of the signal. This gives rise to wavelet transforms which provide better time, frequency localization and multi-resolution analysis of the signal. Wavelet transforms overcomes the STFT limitation by using short windows for higher frequencies and wide windows for lower frequencies, making them as an effective tool in removing noise from signals. In wavelet analysis low frequency coefficients represent the signal \& high frequency coefficients represent noise. Audio signals are non-stationary in nature, therefore analysis of non-stationary signals using Fourier Transform and STFT does not give satisfactory results. The concept of wavelets firstly given by Jean Morlet (Gabor Wavelet) in 1980`s, later on (1984) Goupillaud, Grossmann and Morlet[3] modified the wavelet and introduced the first continuous wavelet transform(CWT). This transforms provide good time, frequency localization. Mallatand Meyer(1988) gives us the concept of multi-resolution in which signal is analyzed by scaling and shifting of wavelet transform. In case of multiresolution, Mallat[4] explained that the wavelet coefficients shown non-Gaussian behavior and narrow peak centered around zero with heavy tailed distributions. Later on Ingrid Daubechies[5] found the compact orthogonal wavelet which requires less time for processing. Afterwards a lot of researchers work on developing fast wavelets transforms which play a great role in signal processing applications. Fischer(1992), Vetterli and Kovaevic(1995), explain the fundamentals of discrete wavelet transform at sub-band level where a signal is separated into two different bands of frequencies. The one of the first application of the DWT is the denoising process, which aims to remove the small part of the signal assumed as noise. While performing noise attenuation, denoising focus on the wavelet shrinkage of the signal. Donoho(1994) and Johnstone(1995) proposed the efficient frequency selective filter approach[6] based on wavelet shrinkage (soft or hard thresholding). Ephraim and Malah[7] also propose the noise suppression rules with recursive noise estimator of priori SNR (signal-to-noise ratio). Further to improve the SNR, element wise thresholding, using a complex wavelet technique proposed.

In our method we use the Additive White Gaussian Noise (AWGN) and Poisson noise which is added linearly to the audio signal. AWGN is random in nature and unrelated means (noise is distributed over the entire frequency spectrum and statically independent). As compared to Gaussian noise, Poisson noise is signaldependent and it is difficult to remove the noise from the signal. Therefore, we use wavelets with Cauchy distribution in removing the noise from signal effectively. Wavelet coefficients are modeled on the basis of the Cauchy distribution. The organization of his paper as follows, Section II presents the theory and methodology 
related to wavelet transforms, Section III give an idea about wavelet thresholding and section IV presents the experimental results. The conclusion is drawn in Section V.

\section{THEORY AND METHODOLOGY}

In this section we first summarize the requisite theory behind the wavelet transforms and Cauchy probability density function. Then, we discuss the effective combination of wavelet thresholding with Cauchy density function to achieve signal-to-noise ratio more accurately and efficiently.

\subsection{Wavelet transforms}

A wave is an oscillating function of time or space and is periodic in nature, whereas wavelets are localized waves and their energy is concentrated in time or space.

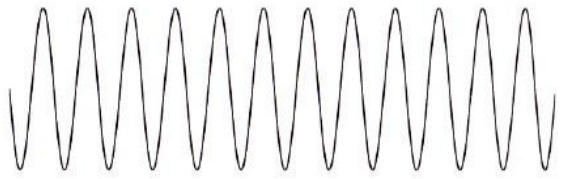

(a)

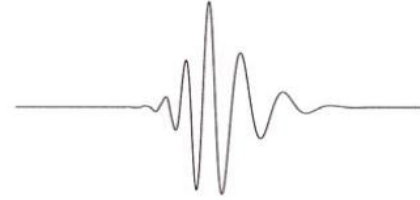

(b)

Figure 1 (a) Wave (b) Wavelet [7]

Compared to the Fourier Transform and STFT, Wavelet Transform uses wavelets of finite energy. The Wavelet Transform provides better time-frequency representation of the signal by using a multi - resolution technique in which different frequencies are analyzed under different resolutions.

The Continuous Wavelet Transform (CWT) is represented by Equation (1), where $\mathrm{x}(\mathrm{t})$ is the signal and $\psi(\mathrm{t})$ is the mother wavelet or the basis function [4/7/9].

$\mathrm{X}_{\mathrm{WT}}(\tau, \mathrm{S})=\frac{1}{\sqrt{|S|}} \int x(t) \cdot \psi *(t-\tau / s) d t$

where ' $\tau$ ' is the translation parameter corresponds to the time information, 's' is the scale parameter relates to the frequency information. The scaling parameter provides detailed information of the signal through large scale and global information of the signal from small scale parameter. A wavelet is represented by two orthogonal functions: the scaling function or father wavelet ' $\phi(t)$ ' \& wavelet function or mother wavelet ' $\psi(t)$ ' $[3 / 10 / 8 / 11]$. The scaling and wavelet functions satisfy,

$\int_{-\infty}^{\infty} \phi(\mathrm{t}) \mathrm{dt}=\mathrm{z}$ and $\int_{-\infty}^{\infty} \psi(\mathrm{t}) \mathrm{dt}=0$

where ' $\mathrm{z}$ ' is a constant and Equation(2) signifies that wavelet function is localized.

The energies of these functions are finite, which means

$\int_{-\infty}^{\infty}|\phi(\mathrm{t})|^{2} d t<\infty$ and $\int_{-\infty}^{\infty}|\psi(\mathrm{t})|^{2} d t<\infty$

The scaling function and the mother wavelet are orthogonal to each other:

$\int_{-\infty}^{\infty} \phi *(\mathrm{t}) \psi(\mathrm{t}) \mathrm{dt}=0$

The complete set of basis function generated by translation and shifting of ' $\phi(t)$ ' and ' $\psi(t)$ '.

\subsection{Discrete Wavelet Transform (DWT)}

Compared to the CWT, DWT provides the sufficient information about the signal and reduces the computation time. In case of CWT, multi-resolution analysis of the signal performed by scaling and translation of the wavelet functions. But DWT is a fast linear operation in which digital filters are used with different cutoff frequencies to analyze time-scale representation of the digital signal. These filter banks decompose the signal into an approximate coefficient (low frequency components) and detailed coefficients (high frequency components). In 1988, S. Mallat represents the scale and translation in power of two also called dyadic scale and translation, which give a better analysis of the signal[8]. DWT represents the length in power of two where wavelet function represents[8/9]

$\psi_{(\mathrm{j}, \mathrm{k})}(\mathrm{x})=2^{\mathrm{j} / 2} \psi\left(2^{\mathrm{j}} \mathrm{x}-\mathrm{k}\right)$

The scaling function is given as

$\phi_{(\mathrm{j}, \mathrm{k})}(\mathrm{x})=2^{\mathrm{j} / 2} \phi\left(2^{\mathrm{j}} \mathrm{x}-\mathrm{k}\right)$

where ' $\psi$ ' is called the wavelet function and ' $j$ ', ' $k$ ' represents the scale index and position respectively. In terms of the wavelet coefficients and scaling coefficients 
$\psi(\mathrm{x})=\sum_{k}^{N-1} G \kappa \sqrt{2 \phi(2 \mathrm{x}-\mathrm{k})}$

where $\mathrm{G}_{0}, \mathrm{G}_{1}, \mathrm{G}_{2} \ldots$ are high pass wavelet coefficients.

$\phi(\mathrm{x})=\sum_{k}^{N-1} H \kappa \sqrt{2 \phi(2 \mathrm{x}-\mathrm{k})}$

where $\mathrm{H}_{0}, \mathrm{H}_{1}, \mathrm{H}_{2} \ldots$ are low pass scaling coefficients.

\subsection{Level of Decomposition}

Multi-resolution analysis uses the filter banks in time-frequency representation of the signal with down-sampling of 2 at each level[10/11].The first stage of the DWT algorithm decomposes the discrete input signal $\mathrm{X}(\mathrm{n})$, into two sets of coefficients. These are the low-frequency scaling coefficients ' $\mathrm{L}_{0}$ ' and highfrequency coefficients ' $\mathrm{H}_{0}$ '. After the filtering process, dyadic decimation [8] or down sampling by a factor of 2 performed at each level, which gives approximated coefficients (low-pass filter decomposition) and detailed coefficients (high-pass filter decomposition). At the end low-pass filter output fed into the filter banks for further decomposition and the process continues till level N.

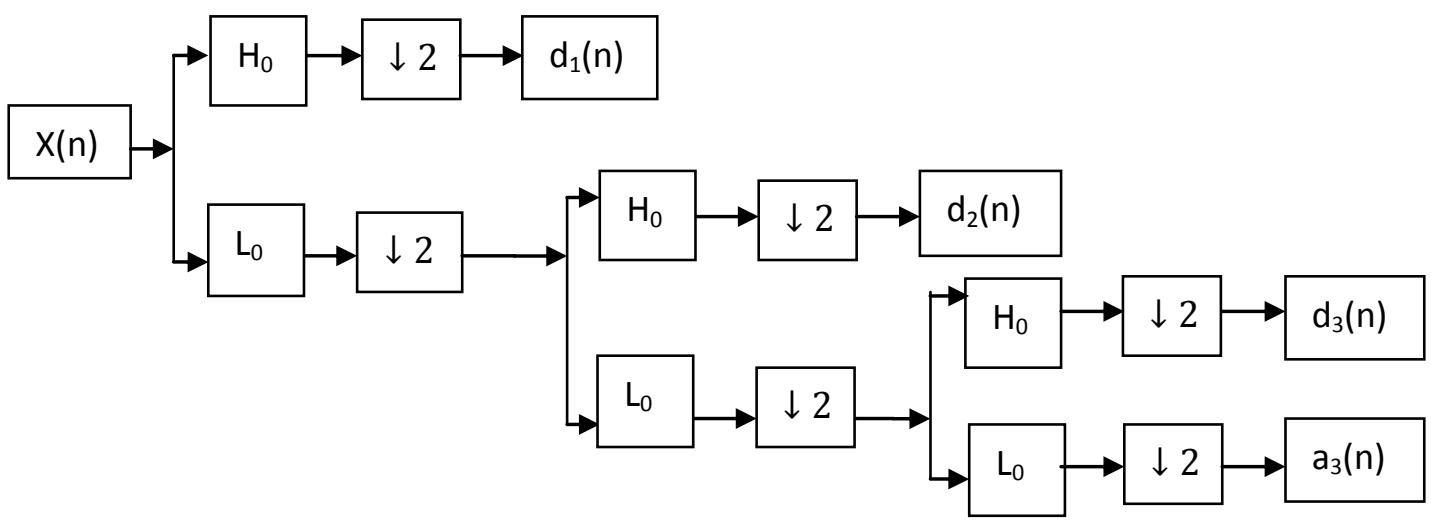

Figure 2 Three level decomposition tree

The original signal can be reconstructed by processing both approximation and detailed coefficients through Upsampling followed by filter banks.

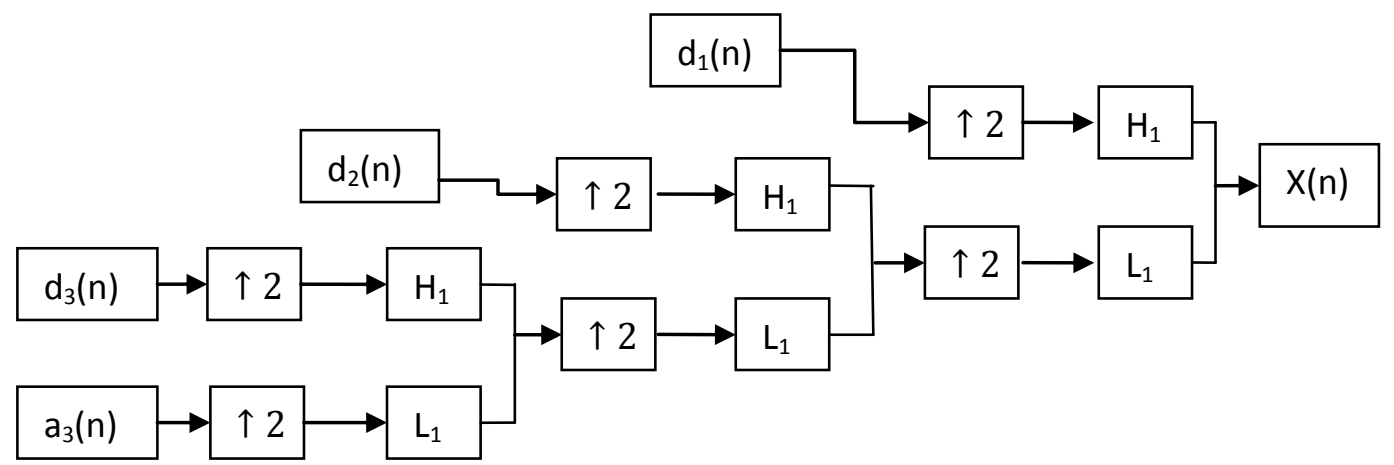

Figure 3 Three level reconstruction tree

\subsection{Cauchy density function}

Mallat and Meyer(1988) give us the concept of multi-resolution in wavelets. In case of multiresolution, Mallat[4] explained that the wavelet coefficients shown non-Gaussian behavior and sharp peak centered around zero with heavy tailed distribution. The Cauchy distribution is a continuous probability density distribution and symmetric therefore its mean is zero [12/13]. But the variance of the Cauchy distribution is infinite, since the integral is unbounded. It is very similar in shape to the normal (Gaussian) distribution. The only difference is that Cauchy tails are much heavier (longer tails) when compared with the normal. So it can be used for non stationary signals as most of the population presents in the extreme values. On the other hand, in the case of finding the objective function and stability, heavy tails perform better than the decreasing tails of 
Gaussian distribution. We use the probabilistic model of heavy-tailed distributions in denoising of an audio signal.

This has the probability density function

$\mathrm{f}(\mathrm{x} ; 0,1)=\frac{1}{\pi\left(1+\mathrm{x}^{2}\right)}$

The Cauchy distribution in terms of location \&scale parameter

$f(x ; a, b)=\frac{1}{\pi b\left(1+\left(\frac{x-a}{b}\right)^{2}\right)}$

where ' $a$ ' is the location parameter, specifying the mean of the distribution, and ' $b$ ' is the scale parameter which specifies the half-width at half-maximum (HWHM)[14].

Its cumulative distribution function

$\mathrm{F}(\mathrm{x} ; 0,1)=\frac{1}{\pi} \arctan (\mathrm{x})+\frac{1}{2}$

In terms of location and scaling parameter

$\mathrm{F}(\mathrm{x} ; \mathrm{a}, \mathrm{b})=\frac{1}{\pi} \arctan \left(\frac{\mathrm{x}-\mathrm{a}}{\mathrm{b}}\right)+\frac{1}{2}$

We use signal to noise ratio as a performance parameter which compares the level of signal with respect to noise level. Signal to noise ratio defined as

$\mathrm{SNR}_{\mathrm{db}}=10 * \log _{10}\left(\frac{\text { Signal }_{\mathrm{db}}}{\text { Noise }_{\mathrm{db}}}\right)$

Noisy_snr explains as the ratio of the original signal to noisy signal.

Noisy_snr $=10 * \log _{10}\left[\frac{\text { norm(signal) }}{\text { norm(signal-noise })}\right]$

denoise $=(\text { noisy }- \text { denoised })^{2}$;

Denoised_snr is the ratio of noisy to denoised signal.

Denoised_snr $=10 * \log _{10}\left[\frac{\left.\text { norm }(\text { (noisy })^{2}\right)}{\text { norm }(\text { denoise })}\right]$

\subsection{De-Noising Method}

For the denoising of the signal it is assumed that the noise energy is distributed in all wavelet coefficients whereas signal energy remains localized to few wavelet coefficients. The idea of thresholding in wavelets was introduced by Donoho and Jonstone[11] and since then wavelet is used in several signal processing applications including removing noise from electrical signals. In this paper, to study the performance of wavelets we use the idea of denoising at different sub band level coefficients. These coefficients are always related to parent node, but individually the non Gaussian behaviour[12]. Therefore for effective denoising we use the combination of wavelets with Cauchy distribution [14] which help us to study about the wavelets. It is assumed that signal power is concentrated in low frequency levels and noise is randomly distributed. The quality of the denoised signal depends upon the good threshold level, wavelet function and decomposition level.

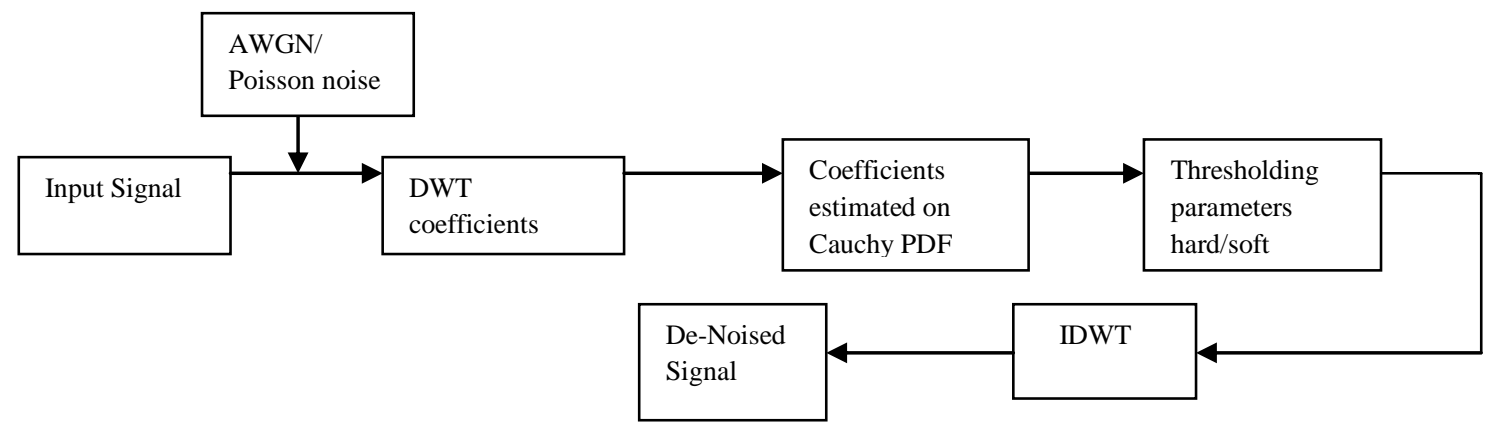

Figure 4 Method of Denoising 


\section{WAVELET THRESHOLDING}

Denoising procedure involves thresholding (Donoho and Johnstone)[15] which helps in filtering of noise as well as retaining the original characteristics of the signal. In wavelet thresholding, signal decomposed into wavelet coefficients and compares the detailed coefficients with the threshold value. Noise is removed from the signal by shrinking these coefficients close to zero. Generally thresholding categorized into two parts: Hard Thresholding and Soft-Thresholding[16].

\begin{tabular}{|c|c|}
\hline "Hard thresholding & Soft thresholding \\
\hline$\eta(x)=\left\{\begin{array}{c}x, \quad|x| \geq T \\
0, \quad \mathrm{x}<T\end{array}\right.$ & $\eta(x)=\left\{\begin{array}{cc}x-T, & x>T \\
0 \quad & |x| \leq T \\
x+T, & x<-T\end{array}\right.$ \\
\hline \multicolumn{2}{|c|}{ where ' $\eta(x)$ ' is the threshold signal, ' $x$ ' is the original signal and ' $T$ ' is the threshold value. } \\
\hline
\end{tabular}

\section{EXPERIMENT \& RESULTS}

We compare the performance of various wavelets in audio signal of duration 13seconds sampled at 44100 samples per second following six wavelet filters, Haar, Db4, Coif5, sym21, biorthogonal 3.5 and Discrete Meyer. Besides this we also analyze the threshold behaviour in denoising of an audio signal. The proposed method for denoising is as follows.

(1) Apply the DWT on the noisy signal, i.e. decomposed into detailed coefficients (cD1) and approximate coefficients (ca1). (2) Cauchy PDF is applied on the wavelet coefficients (cd2, cd3, cd4) of further decomposed levels. (3) Estimate the parameters using equations (10) and (11), respectively. (4) Location parameter ' $a$ ' defined by the average of detailed coefficients and scale parameter ' $\mathrm{b}$ ' is defined by half-width at half-maximum (HWHM). (5) Hard thresholding \& Soft thresholding applied to the detail coefficients of 2, 3, 4 levels. (6) Perform the inverse transform (inverse DWT) of the coefficients obtained in Step 5. (7) For performance analysis SNR value calculated from the audio signal using equations (14) and (15).

\begin{tabular}{|c|c|c|c|c|}
\hline \multicolumn{5}{|c|}{ SIGNAL WITH ADDITION OF 5db ADDITIVE WHITE GAUSSIAN NOISE } \\
\hline Thresholding & & & & \\
\hline$\overline{\text { Wavelets }}$ & "Noisy SNR(db) & "Denoised SNR $(d b)$ & $\begin{array}{ll}\text { Noisy } & S N R(d b)\end{array}$ & "Denoised SNR $(d b)$ \\
\hline Haar & 2.5000 & 0.3372 & 2.4999 & 1.5315 \\
\hline Db4 & 2.5004 & 2.2402 & 2.5001 & 3.6181 \\
\hline Coif5 & 2.5007 & 2.9070 & 2.5003 & 3.6128 \\
\hline Sym 21 & 2.5009 & 3.2960 & 2.5007 & 4.0378 \\
\hline Bior3.5 & 2.5003 & 2.8350 & 2.5002 & 3.7914 \\
\hline dmeyr & 2.4970 & 1.9455 & 2.5008 & 2.3373 \\
\hline
\end{tabular}

Table I

\begin{tabular}{|c|c|c|c|c|}
\hline \multicolumn{5}{|c|}{ SIGNAL WITH ADDITION OF POISSON NOISE } \\
\hline Thresholding & \multicolumn{2}{|c|}{ "HARD } & \multicolumn{2}{|c|}{ SOFT } \\
\hline Wavelets & Noisy $\operatorname{SNR}(d b)$ & "Denoised SNR(db) & Noisy $\quad S N R(d b)$ & Denoised SNR(db) \\
\hline Haar & 1.4649 & 1.4162 & 1.4651 & 2.3543 \\
\hline$D b 4$ & 1.4650 & 3.7790 & 1.4651 & 4.5742 \\
\hline Coif5 & 1.4650 & 2.9690 & 1.4651 & 2.9696 \\
\hline Sym21 & 1.4651 & 4.2146 & 1.4651 & 4.9579 \\
\hline Bior3.5 & 1.4651 & 4.0501 & 1.4651 & 4.6428 \\
\hline dmeyr & 1.4651 & 3.1442 & 1.4651 & 3.6105 \\
\hline
\end{tabular}

Table II

Table $1 \&$ Table II illustrates the SNR values for the various wavelets 

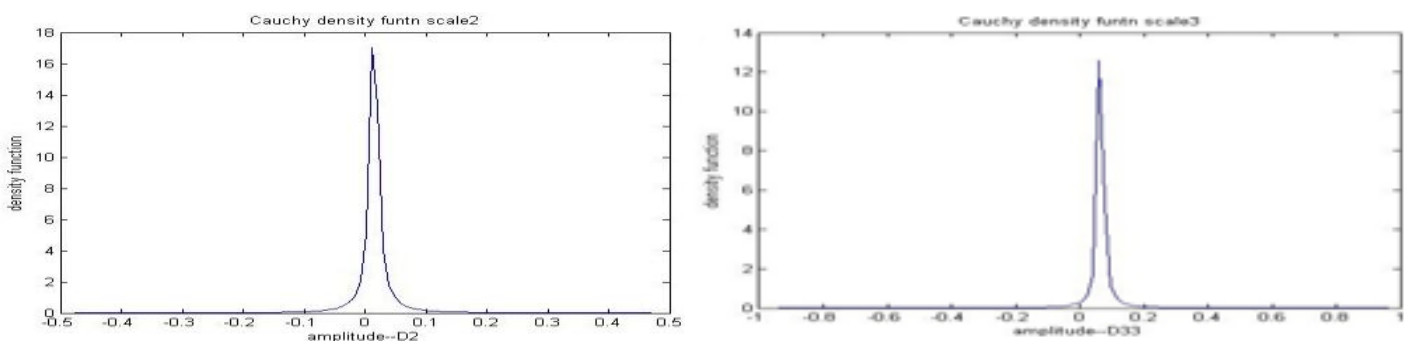

Fig. 5 shows the Cauchy distribution with sharp peak around zero with heavy tailed function

\section{RESULTS:}

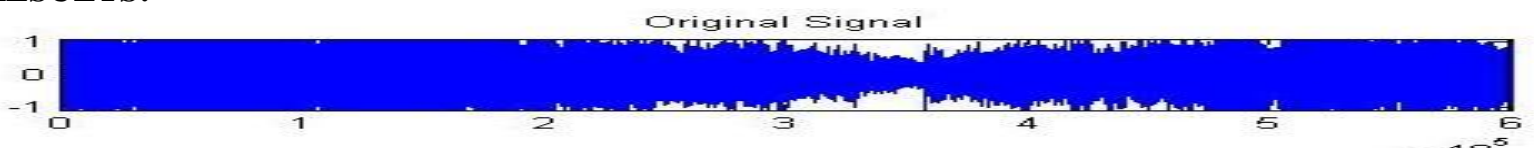

Nais $x$ Signal
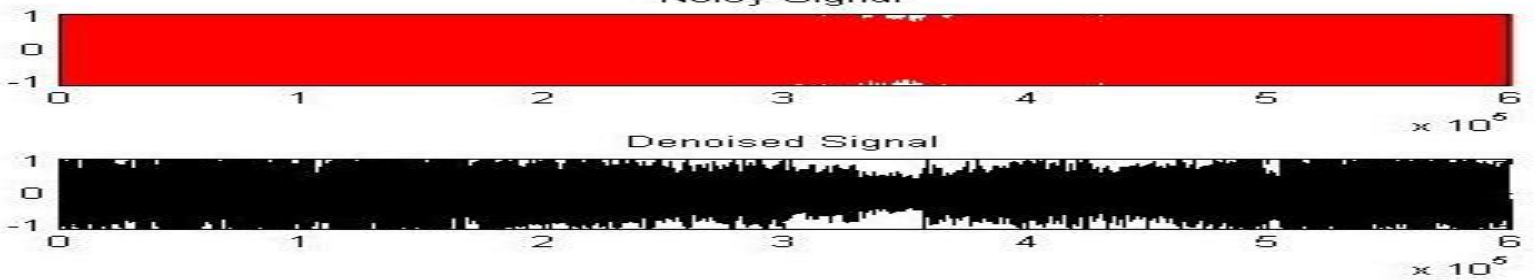

\section{CONCLUSION}

From the Simulation results (Table I \& Table II) it is observed that the performance of haar wavelet is worse than the other wavelets. Sym 21 and coif5 perform better in denoising of an audio signal. Db4 and bior3.5 also shown good results compared to dmeyr. It has been clear that above method shown great results in removing Poisson noise which is more signal dependent. Thresholding plays an important role in achieving high signal to noise values. In case of Soft thresholding we achieved high SNR values and smooth signals than hard thresolding. In overall terms we can conclude that sym21 and coif5 wavelets shown good denoising results and above method is effective in case of removing noise from non-stationary signals.

\section{REFERENCES}

[1] Amara Graps, "An introduction to Wavelets", IEEE Computation Science and Engineering, Vol.2, Issue 2, page 50-61,Jun 1995.

[2] M.Portnoff, "Time-frequency representation of digital signals and systems based on short-time Fourier analysis. Acoustics, Speech and Signal Processing", IEEE Trans on, 1980. 28(1): p. 55-69.

[3] C.S.Burrus,R.A.Gopinath, and H.Guo, "Introduction to Wavelets and Wavelet Tranforms-A Primer". New Jersey, NJ,USA:Prentice Hall, 1997.

[4] S.Mallat, "A theory for multiresolution signal decomposition: the wavelet representation," IEEE Trans. Pattern Anal. Mach. Intell, Vol.11, no. 7, pp. 674-693, Jul. 1989.

[5] Daubechies Ingrid, “Ten Lectures on Wavelets", 9e, SIAM, ISBN: 780-89871-274-2, 2006.

[6] Donoho., Johnston, "De-Noising by Soft Thresholding”, IEEE Trans. on Information Theory, Vol. 41(3), pp. 613-627,1995.

[7] Sachin.P.Nanavati and Prasanta.K.Panigrahi, "Wavelet Transform A New Mathematical microscope", Article, Resonance,March 2004.

[8] Sarita Dangeti, "Denoising Techniques - A Comparison”, A Thesis in partial fulfillment of the requirements for the degree of Master of Science in Electrical Engineering, Louisiana State University,2003.

[9] V. Balakrishnan, Nash Borges, Luke Parchment, "Wavelet Denoising and Speech Enhancement", Spring 2006.

[10] Michael Hazas, "Processing of Non-Stationary Audio Signals" A dissertation for the degree of Master of Philosophy, University of Cambridge, Department of Engineering,31 August 1999.

[11] A. K. Verma, NeemaVerma, "A Comparative Performance Analysis of Wavelets in Denoising of Speech Signals", IJCA Proceedings on National Conference on Advancement of Technologies-Information Systems and Computer Networks, ISCON (1), pp. 29-32,2012.

[12] Xutao Li, Zetao Chen, Shouyong Wang, “An Approximate Representation of Heavy-tailed Noise: Bi-parameter Cauchy-Gaussian Mixture Model”, ICSP Proc.Oct ,2008.

[13] M. I. H. Bhuiyan, M. O. Ahmad, and M. N. S. Swamy, "Spatially Adaptive Wavelet-Based Method Using the Cauchy Prior for Denoising the SAR Images",IEEE Trans. on circuits \& systems for video technology, Vol.17, No. 4, April 2007.

[14] RamchandraManthalkar ,ShubhadaArdhapurkar, SuhasGajre, "Wavelet based ECG denoising by employing Cauchy distribution at subbands", ICSP Proc.Oct ,2008.

[15] Johnston I. M., Silverman B. W., "Wavelet Threshold Estimators for Data with Correlated Noise", Journal of Royal Statistical Society, Series B 59, pp 319-351,1997.

[16] Matlab 7, "Wavelet tool box",http://www.mathworks.com/access/helpdesk/help/toolbox/wavelet/wavelet.shtml. 